\title{
Dielectrophoretic Assembly of High- Density Arrays of Individual Graphene Devices for Rapid Screening
}

\author{
Aravind Vijayaraghavan, ${ }^{\dagger, *}$ Calogero Sciascia, ${ }^{\ddagger \_\S}$ Simone Dehm, ${ }^{\dagger}$ Antonio Lombardo, ${ }^{\S}$ Alessandro Bonetti, ${ }^{\S}$ \\ Andrea C. Ferrari, ${ }^{\S, *}$ and Ralph Krupke ${ }^{\dagger, \perp * *}$ \\ ${ }^{\dagger}$ Institut für Nanotechnologie, Forschungszentrum Karlsruhe, 76021 Karlsruhe, Germany, ${ }^{\ddagger}$ INFM-CNR Physics Department, Politecnico di Milano, Milano, Italy, \\ ${ }^{\S}$ Engineering Department, University of Cambridge, Cambridge CB3 OFA, United Kingdom, and ${ }^{\perp}$ DFG Center for Functional Nanostructures (CFN), 76131 Karlsruhe, \\ Germany
}

I n 2004, graphene, as a free-standing, two-dimensional crystal of carbon atoms, was experimentally shown to exist, ${ }^{1}$ although for the past 60 years it has been theoretically studied as the fundamental structural and electronic building block for various $\mathrm{sp}^{2}$-bonded carbon allotropes such as graphite, ${ }^{2}$ fullerenes, and carbon nanotubes (CNTs). ${ }^{3,4}$ Graphene's promising applications in nanoelectronics ${ }^{5}$ have triggered a rush of research into its production and integration into functional electronic components. Multilayered graphene reaches the 3D limit of graphite in terms of its electronic and dielectric properties at about 10 layers, ${ }^{6,7}$ and therefore the term "few-layer graphene (FLG)" will be used in this paper within this limit. Electronic devices based on graphene can be fabricated either in a top-down approach, where graphene is first grown or deposited on a substrate and subsequently contacted by electrodes, or a bottom-up approach where the desired electrodes are prefabricated on a substrate and graphene subsequently selfassembles or is incorporated at the device locations.

In the top-down approach, the most popular method to generate graphene for research purposes is the micromechanical cleavage of bulk graphite. ' However, this is a low-yield process, where monolayer graphene flakes have to be discerned from a majority of thicker flakes, and is therefore unsuitable for controlled or scaled-up device fabrication. Graphene can also be grown by chemical vapor deposition from hydrocarbon sources on metal substrates ${ }^{8-16}$ or by thermal annealing of SiC. ${ }^{17,18}$ Metal substrates are unsuitable for electronic device applications and require
ABSTRACT We establish the use of dielectrophoresis for the directed parallel assembly of individual flakes and nanoribbons of few-layer graphene into electronic devices. This is a bottom-up approach where source and drain electrodes are prefabricated and the flakes are deposited from a solution using an alternating electric field applied between the electrodes. These devices are characterized by scanning electron microscopy, atomic force microscopy, Raman spectroscopy, and electron transport measurements. They are electrically active and their current carrying capacity and subsequent failure mechanism is revealed. Akin to carbon nanotubes, we show that the dielectrophoretic deposition is self-limiting to one flake per device and is scalable to ultralarge-scale integration densities, thereby enabling the rapid screening of a large number of devices.

KEYWORDS: graphene · dielectrophoresis · directed assembly · bottom-up sample transfer to insulating substrates in order to make useful devices, ${ }^{13,16}$ while the $\mathrm{SiC}$ route inherently limits the substrate choice. An alternative route is to start from graphene solutions. Graphene has been randomly deposited from suspension ${ }^{19}$ on to substrates, the flakes located by AFM or SEM and electrodes fabricated on top to contact them, to yield functional graphene devices. Such a procedure is not easily scalable for controlled device fabrication. Directed assembly of graphene flakes at predetermined locations is thus required.

Here, we demonstrate the fabrication and characterization of electronically active devices of individual FLG flakes using the bottom-up approach, where the flakes are selectively deposited from solution only in between predefined electrodes in a highdensity array using dielectrophoresis.

One approach to obtain graphene solutions involves the dispersion of graphene oxide (GO), ${ }^{20-22}$ stabilized by hydroxyl and epoxide functionalization. GO can be subsequently reduced to graphene, ${ }^{23}$ but leaves a significant number of defects that disrupt the electronic properties. ${ }^{24}$ Recently, much

\footnotetext{
*Address correspondence to v.aravind@int.fzk.de, acf26@eng.cam.ac.uk, krupke@int.fzk.de.
}

Received for review March 23, 2009 and accepted May 25, 2009.

Published online June 10, 2009. 10.1021/nn900288d CCC: $\$ 40.75$ 


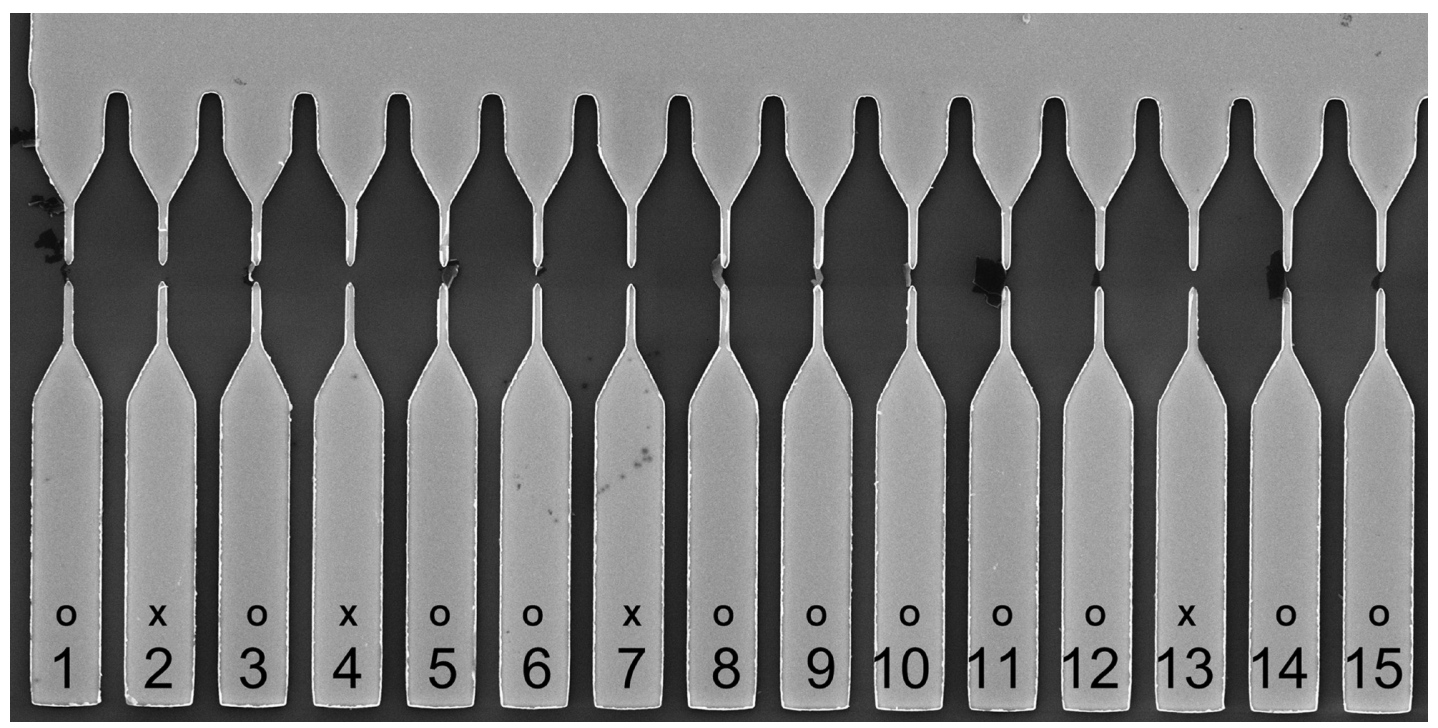

Figure 1. Scanning electron micrograph of a representative region of an array of graphene devices fabricated by dielectrophoretic deposition from a suspension in NMP. Each device comprises a floating source electrode (bottom, labeled 1-15), a common drain electrode (top), and a common back-gate. Eleven out of the 15 devices contain a FLG flake located between the electrode tips. Successfully bridged electrodes are marked as o while nonfunctional devices are marked as x. A zoom in to five of these devices is presented as Supporting Information.

progress has been made in the chemical exfoliation of graphene from bulk graphite. Graphene dispersions, with concentrations of up to $0.01 \mathrm{mg} / \mathrm{mL}$, have been produced by exfoliating graphite in organic solvents such as N-methyl-2-pyrrolidone (NMP). ${ }^{25}$ Graphene dispersions so obtained could be further processed by techniques such as density gradient ultracentrifugation to isolate single-layer and multilayer graphene with high separation yield. Graphene nanoribbons ${ }^{19,26}$ have been fabricated by acid treatment of intercalated expandable graphite and subsequent dispersion and sonication.

Dielectrophoresis has emerged as a powerful technique for the controlled fabrication of nanoelectronic devices. ${ }^{27,28}$ Recently, thin-film devices of GO soot particles $^{29}$ and epitaxial-graphene-GO junctions ${ }^{30}$ have been fabricated by dielectrophoresis. Dielectrophoresis has been applied to separate metallic and semiconducting $\mathrm{CNTs}^{31}$ and used for the fabrication of thin CNT films with controlled alignment and properties. ${ }^{32,33} \mathrm{We}$ have recently demonstrated that individual CNTs can be assembled at ultralarge integration densities into functional electronic devices using dielectrophoresis. ${ }^{34}$ Here, we successfully adopt a similar approach for the fabrication of scalable arrays of functional, individual FLG devices in a three-terminal configuration, although the dimensionality of the FLG flakes is different from that of nanotube or nanowires. This method holds various advantages over other routes for graphene device fabrication and allows for rapid screening of a large number of flakes and devices.

\section{RESULTS AND DISCUSSION}

Figure 1 shows a representative region of the device array, where 11 out of 15 devices are successfully bridged by a flake. A zoom in to five of these devices is shown in Supporting Information. The thickness of the flakes and number of layers is subsequently confirmed by AFM and Raman spectroscopy measurements. We observe flakes of graphite (Figure 2a,b) and FLG (Figure $2 \mathrm{~d}, \mathrm{e}$ ) and FLG nanoribbons (Figure 2g,h). SEM images of other such devices can be found in Supporting Information. SEM shows that suspended graphite and FLG sections have brighter contrast compared to the substrate, while flakes laying flat on the substrate show similar contrast to the substrate irrespective of the number of layers and are identifiable primarily on the basis of edge-contrast. Even within a single flake, regions of different thicknesses can only be discerned in the SEM if the edge-contrast is substantial. SEM imaging was performed at $10 \mathrm{keV}$ acceleration voltage, in order to minimize surface-charging that might perturb the electronic properties of graphene, as has been reported in the case of CNTs. ${ }^{35,36}$ In the absence of charging induced contrast mechanisms, the secondary electron emission coefficients of $\mathrm{SiO}_{2}$ and $\mathrm{C}$ (graphene/ graphite) are nearly identical. ${ }^{37}$ In the case of CNTs, it has been proposed that charging of a suspended CNT in interaction with the electron beam causes large local electric fields around it, which results in an enhanced secondary electron emission. ${ }^{38}$ Similar contrast enhancement or suppression can be obtained by applying an appropriate external bias to the CNT. ${ }^{39}$ In an alternate mechanism, it was reported that a fast electron beam passing through a CNT can generate surface plasmons. ${ }^{40}$ These can excite and accelerate electrons which give an enhanced secondary electron emission probability to the $\mathrm{CNT}^{38}$ and a similar effect might also exist for suspended graphene. Considering the high electron energies, high conductivity of the FLG, and the low contact resistance (as shown later), we propose 
that the latter mechanism is the likely cause for enhanced contrast of suspended flakes.

A high trapping efficiency was obtained with parameters previously optimized for single-wall CNT device assembly. ${ }^{34}$ Further optimization of field frequency and amplitude and graphene concentration is expected to improve the device yield. Note that elongated flakes and nanoribbons assemble with their long axis along the connecting line of the electrodes, as is expected from the induced dipole moment. The quality of the deposited FLG flakes is directly related to the contents of the source suspension. We expect that arrays deposited from a suspension consisting predominantly of singlelayer or bilayer graphene or graphene nanoribbons will give significantly higher yield of devices of the same.

As described earlier, ${ }^{34}$ the density of electrodes on the surface is limited by the thickness of the insulating oxide, and integration densities of 1 million devices per $\mathrm{cm}^{2}$ are obtained. Dielectrophoretic deposition is seen to be self-limiting to one flake or nanoribbon in each device location, because of the higher polarizability of the deposited FLG compared to the surrounding medium. This is similar to previous results with CNTs, and a similar mechanism can also be expected here. When the first such flake or nanoribbon is deposited in the electrode-gap, it changes the dielectrophoretic force fields in its vicinity from attractive to repulsive, thereby limiting further deposition in that electrode gap. In thick graphite devices, with low resistance (shown later), the short-circuiting of the floating electrode with the grounded electrode might also contribute to the selflimiting assembly.

More than 50 flakes were analyzed by Raman spectroscopy. A majority of them consist of multilayer graphene, with some double-layer flakes. We did not find graphene monolayers for the present solutions. On one hand, we expect less than $1 \%$ of the flakes to be monolayers under the sonication conditions used here. ${ }^{25}$ On the other hand, the dielectrophoretic force on the flakes scales proportionally to the volume, and thereby, thicker flakes are deposited preferentially by this process. Figure 3 shows the Raman spectrum, obtained for $514 \mathrm{~nm}$ excitation, of four deposited flakes of increasing number of layers, from bilayer to thick graphite, as evidenced by the shape of the Raman 2D peak. ${ }^{41,42}$ In particular, the four
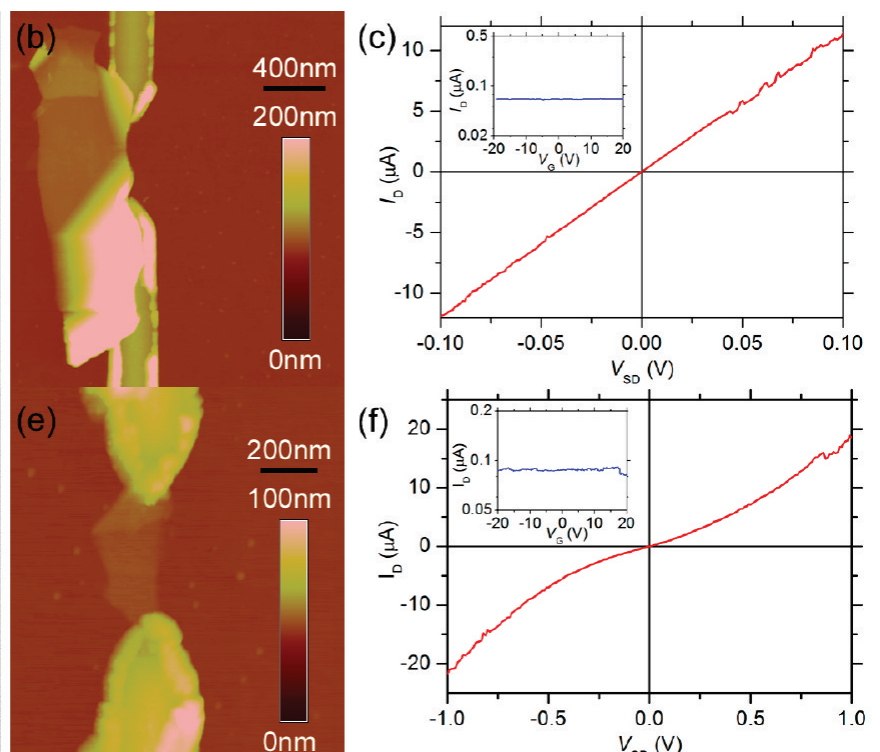

Onm

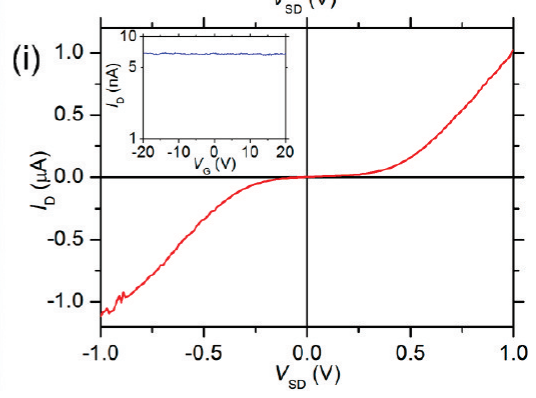

Onm

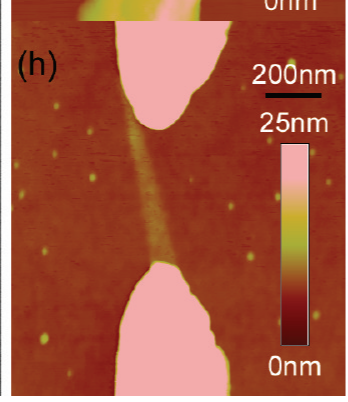

, e, h) atomic force micrograph, and $(c, f, i)$ sion; ( $\mathrm{g}-\mathrm{i})$ thin graphene nanoribbon $(\sim 2$ layers, $\sim 1.5 \mathrm{~nm}$ thick, $\sim 60 \mathrm{~nm}$ wide) showing pronounced low-bias current suppression.

sub-bands are a clear indication of bilayer graphene. ${ }^{42} \mathrm{~A}$ $D$ peak is present, which we attribute to the flake edges due to the smaller size of the flakes compared to the excitation laser spot. ${ }^{41-43}$

Finally, we show that the devices fabricated here are electronically functional. Thick graphite and FLG flakes show linear IV characteristics with low resistances

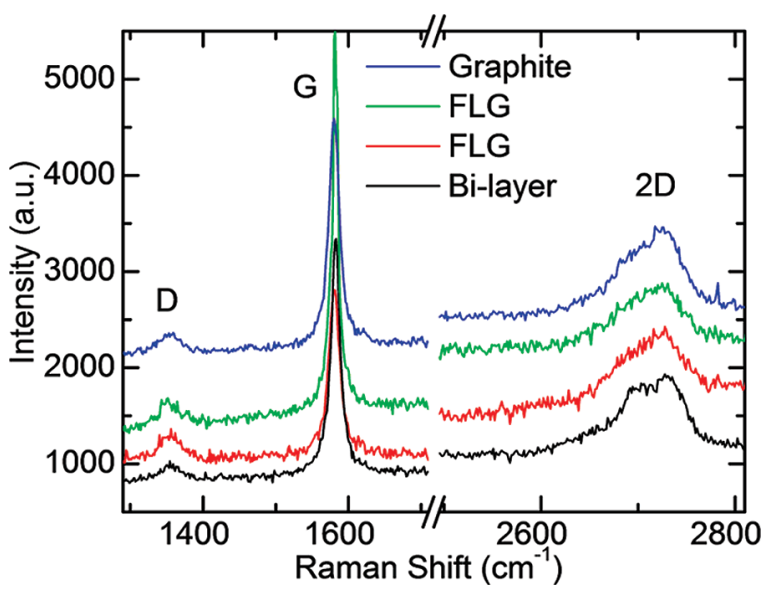

Figure 3. Raman spectra of four flakes. The number of layers is determined by the shape of the $2 \mathrm{D}$ peak, in combination with AFM height measurements.

VOL. 3 - NO. 7 - 1729-1734 - 2009 
of $<10 \mathrm{k} \Omega$ (Figure 2c). Thin FLG flakes have slight nonlinearity at low bias (Figure 2f), while the thin nanoribbon (bilayer) shows a pronounced low-bias current suppression (Figure 2i). The scaling of nonlinearity with the number of layers was previously reported on FLG obtained by reduction of $\mathrm{GO} .{ }^{44}$ This was attributed to conductance suppression in the first graphene layer, owing to interactions with the substrate. The layers would behave as parallel conductors with negligible interlayer conduction, implying that contribution of the first layer diminishes as the flake thickness increases. Raman spectroscopy, however, does not reveal any substrate doping effects in our devices, that is, no upshift and broadening of the $\mathrm{G}$ peak. ${ }^{41,45-47}$ It has also been proposed that when a graphene monolayer is deposited on an oxygen-terminated $\mathrm{SiO}_{2}$ surface, it exhibits a band-gap opening. ${ }^{48}$ This gap reduces as the number of deposited layers increases (as in FLG). Since our $\mathrm{SiO}_{2}$ substrate was subject to oxygen plasma treatment prior to deposition, as required to enable wetting by NMP, the observed nonlinearity could be due to this graphene-substrate interaction. Further investigations, such as deposition of graphene on hydrogenterminated surfaces and the use of other solvents that do not require oxygen plasma treatment of the surface, are currently underway. However, we were unable to detect gate-bias dependence of transconductance in any of the samples. This can be attributed to two factors. The gate-bias window to observe expected ambipolar behavior in graphene was reported to be as high as $\pm 50 \mathrm{~V}$ for $300 \mathrm{~nm}$ thick gate-oxide. ${ }^{1}$ The $800 \mathrm{~nm}$ thick gate-oxide in our devices makes this range even wider due to weaker gate coupling. Also, the charge neutrality point is often shifted beyond the $\pm 20 \mathrm{~V}$ gate range used in our measurements. Because of the presence of unbridged floating electrodes on the surface, which capacitively couple to the back-gate potential, we are unable to explore a gate voltage range wider than $\pm 20 \mathrm{~V}$ without an electric-discharge breakdown between the floating and grounded electrodes.

We note that the resistance of the graphene devices increases upon electron irradiation and decreases after a high-current annealing procedure, ${ }^{49}$ similar to CNT devices. ${ }^{35,36} \mathrm{An}$ increase in resistance of up to 3 orders of magnitude was reported in CNT devices due to the perturbing effect of the high local electric fields arising from charges implanted in the substrate in the vicinity of the nanotube. When these charges are drained through the nanotube under a high bias, the resistance recovers to its original value. However, the changes in current due to electron irradiation and subsequent recovery are substantially smaller (less than an order of magnitude) in graphene compared to CNTs. Detailed investigation of these phenomena in the case of graphene will be presented elsewhere. Our FLG devices were able to sustain high currents, greater than $10 \mu \mathrm{A} /$ layer. This represents current densities of $10^{7} \mathrm{~A} / \mathrm{cm}^{2}$. High-current failure is seen

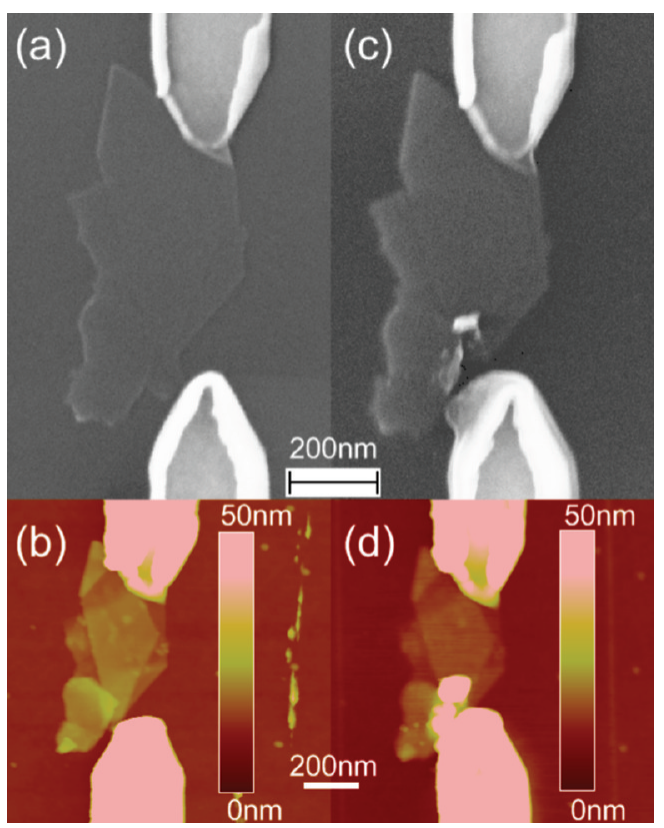

Figure 4. (a, c) SEM and (b, d) AFM of a graphene device (a, b) before and (c, d) after high-current failure. Melting of the metal at the graphene-electrode contact is evident. Supporting Information contains additional such images.

to occur always at the graphene-metal contact, often involving local melting of the metal electrodes, as shown in Figure 4 and Supporting Information. This suggests that the failure mechanism in our devices involves jouleheating of the graphene-metal contact or some other thermally assisted failure mechanism. The electrode melting might also be attributed to electromigration in the narrow $\mathrm{Au}$ - graphene contact region at such high current densities. The region of the FLG flake adjacent to the contact is suspended in most cases and not effectively thermalized by the substrate. It is therefore expected to be the hottest region. It is known in the case of CNTs that the nanotube temperature can exceed the melting point of $\mathrm{Au}\left(1064^{\circ} \mathrm{C}\right)$ at high current $5^{50}$ and a similar mechanism might be in effect in our graphene devices leading to the melting-failure of the electrode. We do not reach the regime of current saturation before failure, where the generation of nonthermalized "hot" phonons would be the dominant failure mechanism, as in CNTs. ${ }^{51}$ The critical current density is also an order of magnitude less than for completely substrate supported graphene, ${ }^{1,52}$ owing to the suspended portion of the FLG flake adjacent to our electrodes.

\section{CONCLUSIONS}

In summary, we have shown that dielectrophoretic deposition enables rapid assembling of individual graphene devices into high-density arrays with high yield. It holds a number of advantages over other methods of graphene device fabrication. Since NMP is used as the solvent the FLG flakes are not coated with any surfactant layer. It is scalable to ultralarge scale integration densities and is self-limiting to one flake or nanoribbon per de- 
vice. It avoids high-temperature processing steps and is compatible with existing microelectronic fabrication technologies. The method is independent of the graphene source and an improvement of the suspensions, in yield and layer selectivity via density gradient ultracentrifugation or similar techniques, could allow the fabrication of high-density arrays of single-layer or bilayer graphene or graphene nanoribbons. The graphene flakes can also be subsequently patterned to form nanoribbons or other branched-ribbon architectures. Such patterning does not require any previous AFM or SEM imaging of the flakes, since their location and the orientation of the electrodes is predefined. Dielectrophoretic deposition of graphene from suspension could emerge as a widely used method for device fabrication for both research and commercial purposes.

\section{MATERIALS AND METHODS}

FLG flakes are dispersed in organic solutions following a similar procedure to that described in ref 22. The starting graphite flakes (NGS Naturgraphit GmbH) have an area of $0.1 \mathrm{~mm}^{2}$ to few $\mathrm{mm}^{2}$. NMP (Sigma-Aldrich) is used as the organic solvent, as it has been found effective in the case of CNTs in forming solutions without a surfactant. ${ }^{53-55}$ A $5 \mathrm{mg}$ portion of graphite is dissolved in $10 \mathrm{~mL}$ of NMP and sonicated for $30 \mathrm{~min}$ followed by centrifugation for $30 \mathrm{~min}$ at $1000 \mathrm{rpm}$ and $20^{\circ} \mathrm{C}$.

FLG devices are fabricated on a substrate of degenerately doped Si with an $800 \mathrm{~nm}$ insulating $\mathrm{SiO}_{2}$ surface layer. The electrodes are first defined by electron-beam lithography and consist of $40 \mathrm{~nm}$ Au over a $5 \mathrm{~nm} \mathrm{Ti}$ adhesion layer. An electrode array design, similar to that for CNTs, ${ }^{34}$ is adopted for FLG devices (Figure 1). It consists of one common drain electrode, which is biased, and an array of floating independent source electrodes, which are not directly connected to the $\mathrm{A} / \mathrm{C}$ source. The alternating electric field is applied between the common drain and conducting Si back-gate. Both source and drain electrodes can also be directly biased; however, this limits the scalability of the process. Instead, all the floating source electrodes capacitively couple to the gate and acquire a similar potential. Prior to deposition, the substrate is rendered hydrophilic by an oxygen plasma treatment to enable the NMP to wet it. A drop $(20 \mu \mathrm{L})$ of suspension is then placed on the substrate and an alternating electric field of $300 \mathrm{kHz}$ and $2 \mathrm{~V} / \mu \mathrm{m}$ is applied for $3 \mathrm{~min}$. The suspension is subsequently removed by a $\mathrm{N}_{2}$ flow.

The devices are imaged by a LEO1530 scanning electron microscope (SEM) and Digital Instruments multimode atomic force microscope (AFM) in tapping-mode to characterize the deposited flakes. The deposited flakes are also characterized by Raman spectroscopy and imaging using a Renishaw and a Witec spectrometer, respectively. Electron transport measurements are performed in situ in the SEM with nanoprobes mounted on Kleindiek Nanotechnik MM3A-EM micromanipulators.

Acknowledgment. The authors acknowledge C. W. Marquardt, M. Engel, J. Coleman, Y. Hernandez, and M. Lotya for helpful discussions. A.V., S.D., and R.K. acknowledge funding by the Initiative and Networking Fund of the Helmholtz-Gemeinschaft Deutscher Forschungszentren (HGF); A.C.F. acknowledges funding from the Royal Society and European Research Council grant NANOPOTS, C.S. from "Fondazione CARIPLO" and Politecnico di Milano, and A.L. from Palermo University.

Supporting Information Available: SEM images of the highdensity array and individual graphene devices at higher magnification and SEM and AFM images of graphene device failure. This material is available free of charge via the Internet at http:// pubs.acs.org.

\section{REFERENCES AND NOTES}

1. Novoselov, K. S.; Geim, A. K.; Morozov, S. V.; Jiang, D.; Zhang, Y.; Dubonos, S. V.; Grigorieva, I. V.; Firsov, A. A. Electric Field Effect in Atomically Thin Carbon Films. Science 2004, 306, 666-669.

2. Wallace, P. R. The Band Theory of Graphite. Phys. Rev. $1947,71,622-634$.

3. lijima, S. Helical Microtubules of Graphitic Carbon. Nature 1991, 354, 56-58.
4. Saito, R.; Fujita, M.; Dresselhaus, G.; Dresselhaus, M. S. Electronic-Structure of Chiral Graphene Tubules. Appl. Phys. Lett. 1992, 60, 2204-2206.

5. Geim, A. K.; Novoselov, K. S. The Rise of Graphene. Nat. Mater. 2007, 6, 183-191.

6. Partoens, B.; Peeters, F. M. From Graphene to Graphite: Electronic Structure Around the K Point. Phys. Rev. B 2006, $74,075404$.

7. Casiraghi, C.; Hartschuh, A.; Lidorikis, E.; Qian, H.; Harutyunyan, H.; Gokus, T.; Novoselov, K. S.; Ferrari, A. C. Rayleigh Imaging of Graphene and Graphene Layers. Nano Lett. 2007, 7, 2711-2717.

8. Land, T. A.; Michely, T.; Behm, R. J.; Hemminger, J. C.; Comsa, G. STM Investigation of Single Layer Graphite Structures Produced on $\mathrm{Pt}(111)$ by Hydrocarbon Decomposition. Surf. Sci. 1992, 264, 261-270.

9. Parga, A. L. V. d.; Calleja, F.; Borca, B.; M, C. G.; Passeggi, Jr.; Hinarejos, J. J.; Guinea, F.; Miranda, R. Periodically Rippled Graphene: Growth and Spatially Resolved Electronic Structure. Phys. Rev. Lett. 2008, 100, 056807.

10. Marchini, S.; Gunther, S.; Wintterlin, J. Scanning Tunneling Microscopy of Graphene on Ru(0001). Phys. Rev. B 2007, $76,075429$.

11. Sutter, P. W.; Flege, J. I.; Sutter, E. A. Epitaxial Graphene on Ruthenium. Nat. Mater. 2008, 7, 406-411.

12. Coraux, J.; N'Diaye, A. T.; Busse, C.; Michely, T. Structural Coherency of Graphene on $\operatorname{Ir}(111)$. Nano Lett. 2008, 8, 565-570.

13. Kim, K. S.; Zhao, Y.; Jang, H.; Lee, S. Y.; Kim, J. M.; Kim, K. S.; Ahn, J.-H.; Kim, P.; Choi, J.-Y.; Hong, B. H. Large-Scale Pattern Growth of Graphene Films for Stretchable Transparent Electrodes. Nature 2009, 457, 706-710.

14. Obraztsov, A. N.; Obraztsova, E. A.; Tyurnina, A. V.; Zolotukhin, A. A. Chemical Vapor Deposition of Thin Graphite Films of Nanometer Thickness. Carbon 2007, 45, 2017-2021.

15. Reina, A.; Jia, X.; Ho, J.; Nezich, D.; Son, H.; Bulovic, V.; Dresselhaus, M. S.; Kong, J. Large Area, Few-Layer Graphene Films on Arbitrary Substrates by Chemical Vapor Deposition. Nano Lett. 2009, 9, 30-35.

16. Li, X.; Cai, W.; An, J.; Kim, S.; Nah, J.; Yang, D.; Piner, R.; Velamakanni, A.; Jung, I.; Tutuc, E.; Banerjee, S. K.; Colombo, L.; Ruoff, R. S. Large-Area Synthesis of HighQuality and Uniform Graphene Films on Copper Foils. Science 2009, 1171245.

17. Berger, C.; Song, Z. M.; Li, T. B.; Li, X. B.; Ogbazghi, A. Y.; Feng, R.; Dai, Z. T.; Marchenkov, A. N.; Conrad, E. H.; First, P. N.; de Heer, W. A. Ultrathin Epitaxial Graphite: 2D Electron Gas Properties and a Route toward GrapheneBased Nanoelectronics. J. Phys. Chem. B 2004, 108, 674-678.

18. Ohta, T.; Bostwick, A.; Seyller, T.; Horn, K.; Rotenberg, E. Controlling the Electronic Structure of Bilayer Graphene. Science 2006, 313, 951-954.

19. Li, X.; Wang, X.; Zhang, L.; Lee, S.; Dai, H. Chemically Derived, Ultrasmooth Graphene Nanoribbon Semiconductors. Science 2008, 319, 1229-1232.

20. Li, D.; Muller, M. B.; Gilje, S.; Kaner, R. B.; Wallace, G. G. Processable Aqueous Dispersions of Graphene Nanosheets. Nat. Nano 2008, 3, 101-105. 
21. Eda, G.; Fanchini, G.; Chhowalla, M. Large-area ultrathin films of reduced graphene oxide as a transparent and flexible electronic material. Nat. Nano 2008, 3, 270-274.

22. Stankovich, S.; Dikin, D. A.; Dommett, G. H. B.; Kohlhaas, K. M.; Zimney, E. J.; Stach, E. A.; Piner, R. D.; Nguyen, S. T.; Ruoff, R. S. Graphene-Based Composite Materials. Nature 2006, 442, 282-286

23. Jung, I.; Dikin, D. A.; Piner, R. D.; Ruoff, R. S. Tunable Electrical Conductivity of Individual Graphene Oxide Sheets Reduced at Low Temperatures. Nano Lett. 2008, 8, 4283-4287.

24. Mkhoyan, K. A.; Contryman, A. W.; Silcox, J.; Stewart, D. A.; Eda, G.; Mattevi, C.; Miller, S.; Chhowalla, M. Atomic and Electronic Structure of Graphene-Oxide. Nano Lett. 2009, 9, 1058-1063.

25. Hernandez, Y.; Nicolosi, V.; Lotya, M.; Blighe, F. M.; Sun, Z.; De, S.; McGovern, I. T.; Holland, B.; Byrne, M.; Gun'Ko, Y. K.; Boland, J. J.; Niraj, P.; Duesberg, G.; Krishnamurthy, S.; Goodhue, R.; Hutchison, J.; Scardaci, V.; Ferrari, A. C.; Coleman, J. N. High-Yield Production of Graphene by Liquid-Phase Exfoliation of Graphite. Nat. Nano 2008, 3, 563-568.

26. Vallés, C.; Drummond, C.; Saadaoui, H.; Furtado, C. A.; He, M.; Roubeau, O.; Ortolani, L.; Monthioux, M.; Pénicaud, A. Solutions of Negatively Charged Graphene Sheets and Ribbons. J. Am. Chem. Soc. 2008, 130, 15802-15804.

27. Liu, Y. L.; Chung, J. H.; Liu, W. K.; Ruoff, R. S. Dielectrophoretic Assembly of Nanowires. J. Phys. Chem. B 2006, 110, 14098-14106.

28. Krupke, R.; Hennrich, F.; Kappes, M. M.; Lohneysen, H. V. Surface Conductance Induced Dielectrophoresis of Semiconducting Single-Walled Carbon Nanotubes. Nano Lett. 2004, 4, 1395-1399.

29. Hong, S.; Jung, S.; Kang, S.; Kim, Y.; Chen, X.; Stankovich, S.; Ruoff, S. R.; Baik, S. Dielectrophoretic Deposition of Graphite Oxide Soot Particles. J. Nanosci. Nanotechnol. 2008, 8, 424-427.

30. Xiaosong, W.; Mike, S.; Xuebin, L.; Fan, M.; Claire, B.; Walt, A. d. H., Epitaxial-Graphene/Graphene-Oxide Junction: An Essential Step towards Epitaxial Graphene Electronics. Phys. Rev. Lett. 2008, 101, 026801.

31. Krupke, R.; Hennrich, F.; von Lohneysen, H.; Kappes, M. M. Separation of Metallic from Semiconducting Single-Walled Carbon Nanotubes. Science 2003, 301, 344-347.

32. Blatt, S.; Hennrich, F.; von Lohneysen, H.; Kappes, M. M.; Vijayaraghavan, A.; Krupke, R. Influence of Structural and Dielectric Anisotropy on the Dielectrophoresis of SingleWalled Carbon Nanotubes. Nano Lett. 2007, 7, 1960-1966.

33. Krupke, R.; Linden, S.; Rapp, M.; Hennrich, F. Thin Films of Metallic Carbon Nanotubes Prepared by Dielectrophoresis. Adv. Mater. 2006, 18, 1468-1470.

34. Vijayaraghavan, A.; Blatt, S.; Weissenberger, D.; Oron-Carl, M.; Hennrich, F.; Gerthsen, D.; Hahn, H.; Krupke, R. UltraLarge-Scale Directed Assembly of Single-Walled Carbon Nanotube Devices. Nano Lett. 2007, 7, 1556-1560.

35. Marquardt, C. W.; Dehm, S.; Vijayaraghavan, A.; Blatt, S.; Hennrich, F.; Krupke, R. Reversible Metal-Insulator Transitions in Metallic Single-Walled Carbon Nanotubes. Nano Lett. 2008, 8, 2767-2772.

36. Vijayaraghavan, A.; Kanzaki, K.; Suzuki, S.; Kobayashi, Y.; Inokawa, H.; Ono, Y.; Kar, S.; Ajayan, P. M. MetalSemiconductor Transition in Single-Walled Carbon Nanotubes Induced by Low-Energy Electron Irradiation. Nano Lett. 2005, 5, 1575-1579.

37. Seiler, H. Secondary Electron Emission in the Scanning Electron Microscope. J. Appl. Phys. 1983, 54, R1-R18.

38. Kasumov, Y. A.; Khodos, I. I.; Kociak, M.; Kasumov, A. Y. Scanning and Transmission Electron Microscope Images of a Suspended Single-Walled Carbon Nanotube. Appl. Phys. Lett. 2006, 89, 013120.

39. Vijayaraghavan, A.; Blatt, S.; Marquardt, C.; Dehm, S.; Wahi, R.; Hennrich, F.; Krupke, R. Imaging Electronic Structure of Carbon Nanotubes by Voltage-Contrast Scanning Electron Microscopy. Nano Res. 2008, 1, 321-332.
40. Stéphan, O.; Taverna, D.; Kociak, M.; Suenaga, K.; Henrard, L.; Colliex, C. Dielectric Response of Isolated Carbon Nanotubes Investigated by Spatially Resolved Electron Energy-Loss Spectroscopy: From Multiwalled to SingleWalled Nanotubes. Phys. Rev. B 2002, 66, 155422.

41. Ferrari, A. C. Raman Spectroscopy of Graphene and Graphite: Disorder, Electron-Phonon Coupling, Doping and Nonadiabatic Effects. Solid State Commun. 2007, 143, 47-57.

42. Ferrari, A. C.; Meyer, J. C.; Scardaci, V.; Casiraghi, C.; Lazzeri, M.; Mauri, F.; Piscanec, S.; Jiang, D.; Novoselov, K. S.; Roth, S.; Geim, A. K. Raman Spectrum of Graphene and Graphene Layers. Phys. Rev. Lett. 2006, 97, 187401.

43. Casiraghi, C.; Hartschuh, A.; Qian, H.; Piscanec, S.; Georgi, C.; Novoselov, K. S.; Basko, D. M.; Ferrari, A. C. Raman Spectroscopy of Graphene Edges. Nano Lett. 2009, 9, 1433-1441.

44. Gomez-Navarro, C.; Weitz, R. T.; Bittner, A. M.; Scolari, M.; Mews, A.; Burghard, M.; Kern, K. Electronic Transport Properties of Individual Chemically Reduced Graphene Oxide Sheets. Nano Lett. 2007, 7, 3499-3503.

45. Pisana, S.; Lazzeri, M.; Casiraghi, C.; Novoselov, K. S.; Geim, A. K.; Ferrari, A. C.; Mauri, F. Breakdown of the Adiabatic Born-Oppenheimer Approximation in Graphene. Nat. Mater. 2007, 6, 198-201.

46. Das, A.; Pisana, S.; Chakraborty, B.; Piscanec, S.; Saha, S. K.; Waghmare, U. V.; Novoselov, K. S.; Krishnamurthy, H. R.; Geim, A. K.; Ferrari, A. C.; Sood, A. K. Monitoring Dopants by Raman Scattering in an Electrochemically Top-Gated Graphene Transistor. Nat. Nanotechnol. 2008, 3, 210-215.

47. Das, A.; Chakraborty, B.; Piscanec, S.; Pisana, S.; Sood, A. K.; Ferrari, A. C. Phonon Renormalisation in Doped Bilayer Graphene. Phys. Rev. B 2009, 79, 155417.

48. Shemella, P.; Nayak, S. K. Electronic Structure and BandGap Modulation of Graphene via Substrate Surface Chemistry. Appl. Phys. Lett. 2009, 94, 032101.

49. Moser, J.; Barreiro, A.; Bachtold, A. Current-Induced Cleaning of Graphene. Appl. Phys. Lett. 2007, 91, 163513.

50. Begtrup, G. E.; Ray, K. G.; Kessler, B. M.; Yuzvinsky, T. D.; Garcia, H.; Zettl, A. Probing Nanoscale Solids at Thermal Extremes. Phys. Rev. Lett. 2007, 99, 155901.

51. Lazzeri, M.; Piscanec, S.; Mauri, F.; Ferrari, A. C.; Robertson, J. Electron Transport and Hot Phonons in Carbon Nanotubes. Phys. Rev. Lett. 2005, 95, 236802.

52. Meric, I.; Han, M. Y.; Young, A. F.; Ozyilmaz, B.; Kim, P.; Shepard, K. L. Current Saturation in Zero-Bandgap, TopGated Graphene Field-Effect Transistors. Nat. Nanotechnol. 2008, 3, 654-659.

53. Giordani, S.; Bergin, S. D.; Nicolosi, V.; Lebedkin, S.; Kappes, M. M.; Blau, W. J.; Coleman, J. N. Debundling of SingleWalled Nanotubes by Dilution: Observation of Large Populations of Individual Nanotubes in Amide Solvent Dispersions. J. Phys. Chem. B 2006, 110, 15708-15718.

54. Bergin, S. D.; Nicolosi, V.; Streich, P. V.; Giordani, S.; Sun, Z.; Windle, A. H.; Ryan, P.; Niraj, N. P. P.; Wang, Z.-T. T.; Carpenter, L.; Blau, W. J.; Boland, J. J.; Hamilton, J. P.; Coleman, J. N. Towards Solutions of Single-Walled Carbon Nanotubes in Common Solvents. Adv. Mater. 2008, 20, 1876-1881.

55. Hasan, T.; Scardaci, V.; Tan, P. H.; Rozhin, A. G.; Milne, W. I.; Ferrari, A. C. Stabilization and "Debundling" of Single-Wall Carbon Nanotube Dispersions in N-Methyl-2pyrrolidone (NMP) by Polyvinylpyrrolidone (PVP). J. Phys. Chem. C 2007, 111, 12594-12602. 